\title{
Adherence to the Spanish dietary guidelines and its association with obesity in the European Prospective Investigation into Cancer and Nutrition (EPIC)-Granada study
}

\author{
Esther Molina-Montes ${ }^{1,2}$, Irina Uzhova ${ }^{3}$, Elena Molina-Portillo ${ }^{1}$, José-María Huerta ${ }^{2,4}$, \\ Genevieve Buckland ${ }^{5}$, Pilar Amiano ${ }^{2,6}$, Virginia Menéndez ${ }^{7}$, Aurelio Barricarte ${ }^{2,8}$, \\ Carlos A González ${ }^{5}$ and María-José Sánchez ${ }^{1,2, *}$ \\ ${ }^{1}$ Andalusian School of Public Health, Granada Cancer Registry, Instituto de Investigación Biosanitaria de Granada \\ (Granada.bs), Campus Universitario de Cartuja, Cuesta del Observatorio 4, E-1 8080 Granada, Spain: ${ }^{2} \mathrm{CIBER}$ de \\ Epidemiología y Salud Pública (CIBERESP), Madrid, Spain: ${ }^{3}$ Department of Bioscience and Nutrition, Karolinska \\ Institute, Stockholm, Sweden: ${ }^{4}$ Department of Epidemiology, Murcia Regional Health Council, Murcia, Spain: ${ }^{5}$ Unit \\ of Nutrition, Environment and Cancer, Cancer Epidemiology Research Programme, Catalan Institute of Oncology \\ (ICO-IDIBELL), Barcelona, Spain: 'Public Health Division of Gipuzkoa, BioDonostia Research Institute, San Sebastián, \\ Spain: ${ }^{7}$ Public Health Directorate, Asturias, Spain: ${ }^{8}$ Public Health Institute of Navarra, Pamplona, Spain
}

Submitted 21 October 2013: Final revision received 10 March 2014: Accepted 24 March 2014: First published online 24 April 2014

\begin{abstract}
Objective: Dietary guidelines are intended to prevent chronic diseases and obesity. The aim of the present study was to develop a diet quality index based on the Spanish Food Pyramid (SFP) and to further explore its association with obesity in the European Prospective Investigation into Cancer and Nutrition (EPIC)Granada study.

Design: Cross-sectional study. The SFP score considered recommendations given for twelve food groups, and for physical activity and alcohol consumption. Obesity was defined as BMI over $30 \mathrm{~kg} / \mathrm{m}^{2}$ and abdominal obesity as waist circumference larger than $102 \mathrm{~cm}$ (men) and $88 \mathrm{~cm}$ (women). Logistic regression was conducted to estimate odds ratios of obesity by quintiles and by 10-point increment in adherence to the score, controlling for potential confounders.

Setting: EPIC-Granada study.

Subjects: Participants ( $n$ 6717) aged 35-69 years ( $77 \%$ women).

Results: A 10-point increase in adherence to the SFP score was associated with a $14 \%(\mathrm{OR}=0 \cdot 86 ; 95 \%$ CI $0.79,0.94)$ lower odds of obesity in men ( $P$ interaction by sex $=0 \cdot 02)$. The odds of abdominal obesity decreased globally by $12 \%(\mathrm{OR}=0 \cdot 88$; $95 \%$ CI $0.84,0.93)$ per 10 -point increase in adherence to this score. The effect of higher adherence to the score on abdominal obesity was stronger in physically inactive men and women $\left(\mathrm{OR}_{\text {per }}\right.$ 10-point increase $=0.79 ; 95 \% \mathrm{CI} 0.68,0.92$ and $\mathrm{OR}_{\text {per 10-point increase }}=0 \cdot 89 ; 95 \%$ CI 0.84, 0.95, respectively).

Conclusions: These findings support that the Spanish dietary guidelines might be an effective tool for obesity prevention. However, prospective studies investigating this association are warranted.
\end{abstract}

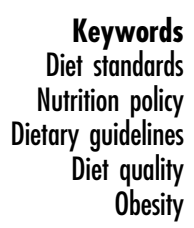

Overweight and obesity is known to be a worldwide problem that has tripled in many countries within the European region since the 1980s, along with the burden of several chronic diseases linked to this major risk factor ${ }^{(1)}$. Estimates and trends show that its prevalence will continue to rise within the next decade ${ }^{(1,2)}$. The obesity epidemic is of great concern in Spain due to the steady increase in prevalence; currently $23 \%$ of the population is estimated to be obese ${ }^{(3)}$. Since obesity is linked to dietary habits and physical inactivity, prevention through dietary and other lifestyle interventions is nowadays recognized as a major priority ${ }^{(1)}$.

The emerging scientific evidence on diet-disease relationships has expanded the traditional focus on nutrient adequacy to the development of dietary guidelines that are intended to promote a healthy diet and to reduce the risk of chronic diseases and obesity ${ }^{(4)}$. In Spain the first nutritional objectives and dietary recommendations were 
developed by the Spanish Society of Community Nutrition (SENC) in $1994^{(5)}$. Food-based dietary guidelines, the so-called 'Spanish Food Pyramid' (SFP), were developed along with the nutritional objectives in $2001^{(6)}$ and subsequently revised in $2004^{(7)}$, including recommendations on portion sizes and frequencies of consumption of specific food groups. The nutritional objectives for the Spanish population were also recently updated after the agreement reached by the SENC in $2011^{(8)}$.

The way to evaluate the dietary guidelines is to assess whether compliance to them leads to health benefits ${ }^{(9)}$. For this purpose, some studies have transferred them into a diet quality index by scoring the individual's diet with a set of criteria of dietary quality, which can later on be related to the health outcome ${ }^{(9-11)}$. In general, the indices are based either on the recommendations of dietary guidelines (Healthy Eating Index, Diet Quality Index, etc.) or on a defined dietary pattern (Mediterranean diet score, etc. $)^{(9,12)}$.

The majority of studies have investigated the relationship between compliance to dietary guidelines and the risk of chronic diseases ${ }^{(10,11,13)}$. However, only a few have focused on obesity risk as an outcome ${ }^{(14-19)}$. The Healthy Eating Index (HEI) has been evaluated in relation to obesity risk in a prospective ${ }^{(14)}$ study and in a crosssectional study ${ }^{(15)}$, showing that this index is an important predictor of obesity. A higher adherence to a revised version of the Diet Quality Index (DQI) was also associated with a lower obesity risk in a prospective study ${ }^{(16)}$. Within Europe, the French Programme National Nutrition Santé-Guideline Score (PNNS-GS), among other diet quality indices, was examined in the SU.VI.MAX (SUpplementation en VItamines et Minéraux AntioXydants study) cohort, revealing a significant reduction in obesity risk after 6 years ${ }^{(17)}$ and 13 years of follow-up ${ }^{(18)}$. Also the Finnish Diet Score (FDS), based on the country's dietary guidelines, has been shown to be inversely associated with obesity ${ }^{(19)}$.

Most countries, including Spain, have not yet developed a scoring system to address the diet quality of their dietary guidelines; therefore there is not enough evidence yet to determine whether adherence to these guidelines prevents obesity. We aimed to develop a diet quality index (the Spanish Food Pyramid Score) to assess the adherence to the guidelines and to further explore its relationship with obesity in the EPIC (European Prospective Investigation into Cancer and Nutrition)-Granada study.

\section{Methods}

\section{Study population}

Details of the design and methodology of the EPIC-Spain study have been described elsewhere ${ }^{(20,21)}$. In brief, EPICSpain is part of the EPIC study, aimed at investigating the relationship between diet and cancer. The EPIC study includes over half a million participants; approximately 41000 were recruited from the five centres of Spain: Asturias, Navarra and San Sebastian in the North, Murcia in the South-East and Granada in the South ${ }^{(21)}$. The EPICGranada cohort includes 7879 adults aged 35-69 years (77\% women), mainly blood donors, recruited between 1992 and 1996 from the province of Granada. One hundred and fifty-six individuals with extreme values of energy intake $(<1 \%$ and $>99 \%$ percentile of energy intake, i.e. below $3105 \mathrm{~kJ} / \mathrm{d}(742 \mathrm{kcal} / \mathrm{d})$ and above $16209 \mathrm{~kJ} / \mathrm{d}$ (3874 kcal/d), respectively), 149 individuals with missing values in the variables of interest and 857 individuals with self-reported diseases at recruitment (cancer, diabetes or CVD) were excluded. After these exclusions 6717 participants remained for the data analysis. Approval for the study was obtained from the ethical review boards of the International Agency for Research on Cancer and specifically for EPIC-Spain from the Medical Ethical Committee of Bellvitge Hospital. All participants provided written informed consent ${ }^{(21)}$.

\section{Dietary assessment}

Information on diet was collected through a validated diet history questionnaire ${ }^{(22,23)}$. The questionnaire was administered through personal interviews asking participants about the frequency of consumption and portion sizes of more than 600 food items, including more than 200 recipes and regional dishes, during the preceding 12 months. Seasonal differences in food intake and variations during the weekends were also taken into account. Additional questions about added fat and its type used for food preparation, and about intake of alcoholic beverages, were included $^{(22)}$. Individuals' energy and nutrient intakes were derived through a standardized nutrient database ${ }^{(24)}$.

\section{The Spanish dietary guidelines score}

Adherence to the Spanish dietary guidelines was assessed taking the 2004 revised version of the Spanish Food Pyramid Score (SFP score) ${ }^{(7)}$. This SFP includes recommendations for the following food groups: potatoes, pasta, rice and bread, water, vegetables, fruits, milk and dairy products, olive oil, fish, lean meat, poultry and eggs, legumes, nuts, meat products and fatty meat, sweets, snacks, butter and margarine. Recommendations are also given for physical activity and moderate wine and beer consumption. Adherence was assessed using the method proposed for the computation of the German Food Pyramid Index ${ }^{(13)}$. Details of the SFP score computation are shown in Table 1. In brief, intake of food groups was first adjusted for energy intake by using the energy density method (g/8368 kJ (2000 kcal)). The number of servings consumed, the reported frequency of consumption, was calculated by dividing the intake of each food group $(\mathrm{g} / \mathrm{d}$ ) by the recommended serving size as defined in the SFP. The score for food groups recommended on a daily or 
Table 1 The Spanish Food Pyramid and the developed score (SFP score) of adherence to the Spanish dietary guidelines (SENC-2004)

\begin{tabular}{|c|c|c|c|c|}
\hline Food groups/subgroups* & Serving size $(\mathrm{g})^{*}$ & Servings ${ }^{*}$ & Operationalization $\dagger$ & Scoring \\
\hline $\begin{array}{l}\text { Potatoes (potatoes and tubers) } \\
\text { Rice, pasta, flours and cereals (flour, semolina, starches, flakes, pasta, rice, grains, breakfast cereals) } \\
\text { Bread (bread, crispbread, rusks, dough) }\end{array}$ & $\begin{array}{l}150-200 \\
60-80 \\
40-60\end{array}$ & $4-6$ per $d$ & $\begin{array}{l}\text { Equation (1) if servings } \leq 6 \\
\text { Equation (2) if exceeded }\end{array}$ & $0-10$ \\
\hline $\begin{array}{l}\text { Whole mealf (bread, crispbread, rusks and pasta, semolina, starches, flakes, pasta, rice, } \\
\text { grains, breakfast cereals) }\end{array}$ & & & †lf whole meal & 10 \\
\hline $\begin{array}{l}\text { Vegetablesł (leafy, fruiting and root vegetables, cabbages, mushrooms, stalk, grain and pod } \\
\text { vegetables, onion and garlic, mixed vegetables) }\end{array}$ & $150-200$ & $\geq 2$ per $d$ & $\begin{array}{l}\text { Equation (1) if servings } \geq 2 \\
\text { ¥lf exceeded }\end{array}$ & $\begin{array}{l}0-10 \\
10\end{array}$ \\
\hline Fruits $\ddagger$ (all types of fruits: stone fruits, citrus fruits, berries, banana, grapes, etc.) & $120-200$ & $\geq 3$ per $d$ & $\begin{array}{l}\text { Equation (1) if servings } \geq 3 \\
\text { ¥lf exceeded }\end{array}$ & $\begin{array}{c}0-10 \\
10\end{array}$ \\
\hline Olive oil (olive oil) & 10 & $3-6$ per d & $\begin{array}{l}\text { Equation (1) if servings } \leq 6 \\
\text { Equation (2) if exceeded }\end{array}$ & $0-10$ \\
\hline Milk and yoghurt (milk and milk beverages, yoghurt, milk-based desserts) & 200-250 each & $3-4$ per d & $\begin{array}{l}\text { Equation }(1) \text { if servings } \leq 4 \\
\text { Equation }(2) \text { if exceeded }\end{array}$ & $0-10$ \\
\hline $\begin{array}{l}\text { Mature cheese (all types of cheese except fresh) } \\
\text { Fresh cheese (curd and ricotta type cheese) }\end{array}$ & $\begin{array}{c}40-60 \\
125\end{array}$ & & & \\
\hline Fish (all types of fish, including shellfish) & $125-150$ & 3-4 per week & $\begin{array}{l}\text { Equation (1) if servings } \leq 0 \cdot 6 \\
\text { Equation (2) if exceeded }\end{array}$ & $0-10$ \\
\hline Lean meat§ (rabbit, horse, goat, veal), poultry (chicken, turkey), eggs (eggs and egg products) & $100-125$ & 3-4 per week & $\begin{array}{l}\text { Equation (1) if servings } \leq 0.6 \\
\text { Equation (2) if exceeded }\end{array}$ & $0-10$ \\
\hline Legumes (legumes) & $60-80$ & 3-4 per week & $\begin{array}{l}\text { Equation (1) if servings } \leq 0.6 \\
\text { Equation (2) if exceeded }\end{array}$ & $0-10$ \\
\hline Nuts (tree nuts, peanuts, etc. and seeds) & $20-30$ & 3-7 per week & $\begin{array}{l}\text { Equation (1) if servings } \leq 1 \\
\text { Equation (2) if exceeded }\end{array}$ & $0-10$ \\
\hline $\begin{array}{l}\text { Processed meat \& sausages (meat products) (bacon, ham, offal, hamburger, meatballs, minced } \\
\text { meat, sausages) }\end{array}$ & 60 & Occasionally & $\begin{array}{l}\text { Equation (2) if servings } \leq 0.3(0.15 \\
\text { servings of each) } \\
\text { Equation (1) if exceeded }\end{array}$ & $0-10$ \\
\hline $\begin{array}{l}\text { Fatty meat§ (beef, pork, lamb, duck, goose, game) } \\
\text { Sweets (sugar, honey, candy bars, paste, confectionery, ice cream, syrup, sorbet) }\end{array}$ & $\begin{array}{l}80 \\
50\end{array}$ & Occasionally & $\begin{array}{l}\text { Equation (2) if servings } \leq 0.3(0.15 \\
\text { servings of each) }\end{array}$ & $0-10$ \\
\hline $\begin{array}{l}\text { Snacks (salty biscuits, crackers, aperitif biscuits) } \\
\text { Sugary soft drinks\|l (soft drinks, juices \& nectars) } \\
\text { Cakes (cakes, biscuits, pastries, puddings) }\end{array}$ & $\begin{array}{l}50 \\
330 \\
150\end{array}$ & & Equation (1) if exceeded & \\
\hline Butter (butter) & 10 & Occasionally & $\begin{array}{l}\text { Equation (2) if servings } \leq 0.3(0.15 \\
\text { servings of each) }\end{array}$ & $0-10$ \\
\hline Margarine (margarine) & & & Equation (1) if exceeded & \\
\hline 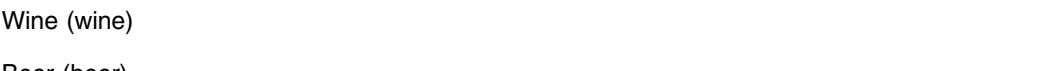 & $100 \mathrm{ml}$ or & $\begin{array}{l}\text { Optional per } \\
\text { day }\end{array}$ & $\begin{array}{l}\text { Non-drinkers and drinkers below } \\
\text { recommendation levels }\end{array}$ & 10 \\
\hline $\begin{array}{l}\text { Beer (beer) } \\
\text { Physical activity } 9 \text { (MET hour of walking) }\end{array}$ & $\begin{array}{l}200 \mathrm{ml} \\
\geq 30 \mathrm{~min}\end{array}$ & Every day & $\begin{array}{l}\text { Drinkers if exceeded intake } \\
0-<15 \mathrm{~min} / \mathrm{d} \\
15-<30 \mathrm{~min} / \mathrm{d}\end{array}$ & $\begin{array}{l}0 \\
5 \\
10\end{array}$ \\
\hline Water** & $200 \mathrm{ml}$ & $4-8$ per d & $\begin{array}{l}\geq 30 \mathrm{~min} / \mathrm{d} \\
\text { No information available }\end{array}$ & 10 \\
\hline
\end{tabular}

SENC, Spanish Society of Community Nutrition; MET, metabolic equivalents; EPIC, European Prospective Investigation into Cancer and Nutrition.

${ }^{*}$ Food groups and subgroups, serving sizes and servings per day as described by the SFP, except for occasionally recommended food groups for which serving sizes were not defined. Standard portion sizes have been defined for these food groups. Daily serving size for recommendations on a weekly basis = maximum serving per week/7 d. 'Occasionally' was considered as 2 times per week.

†Number of servings consumed = intake of each food group ( $\mathrm{g} / \mathrm{d})$ /recommended serving size (mid-point of the serving size range). For intakes agreeing with recommendations, equation (1): score = servings (consumed)/ servings (recommended) $\times 10$. For intakes exceeding recommendations, equation (2): score $=$ servings (recommended)/servings $($ consumed) $\times 10$. Non-consumers get 10 points in the case of occasionally recommended food groups.

$\$ 10$ extra points were given if whole-meal products (bread, crispbread and rusks) were consumed within the recommendations, and if consumption of fruits and vegetables exceeded the recommendations of 3 and 2 servings/d, respectively.

§Lean meat and fatty meat were considered as below or over $10 \mathrm{~g}$ fat $/ 100 \mathrm{~g}$ edible portion, respectively.

IIFruits and vegetables juices, concentrates and nectars (i.e. soft drinks, juices with added sugars) were considered 'Sugary soft drinks'.

IDespite physical activity not being a dietary component of the SFP, it has been considered for the SFP score operationalization because it is another component of the SFP.

${ }^{*}$ Information on water was unavailable in the EPIC-Granada cohort and was therefore not considered for the SFP score operationalization. 
weekly basis (potatoes, rice, bread, whole meal as bread and pasta, fruits and vegetables, olive oil, fish, lean meat, poultry, eggs, legumes and nuts) was calculated using the following equation: score $=$ number of servings consumed/servings recommended $\times 10$, allowing up to 10 extra points if recommendations for fruits and vegetables were exceeded. In addition, 10 extra points were given to those who preferred consumption of whole-meal products (bread, crispbread, rusks and pasta) because the SFP sets this as another recommendation. For intakes exceeding the recommended servings, except for fruits and vegetables, the inversed equation was used: score $=$ servings recommended/servings consumed $\times 10$. The score for food groups recommended as occasional consumption (sweets, snacks, butter, soft drinks, meat products and fatty meats) was calculated considering the same equations, but in a reversed manner. A maximum score of 10 points was assigned if intake did not exceed the recommended serving. Non-drinkers of alcohol at recruitment and participants who drank beer or wine within the permitted range got 10 points, while those who exceeded the maximum levels got 0 points. For physical activity recommendations (walking more than $30 \mathrm{~min}$ every day), values of 0 points (less than $15 \mathrm{~min}$ ), 5 points (15 to $30 \mathrm{~min}$ ) and 10 points (more than $30 \mathrm{~min}$, equivalent to 10.5 MET (metabolic equivalents) hour of walking) were assigned. The SFP also sets a recommendation for water consumption that was not operationalized into the SFP score because this information was unavailable, i.e. the dietary questionnaire used at recruitment of the EPIC participants did not account for intake of water.

Each component of the SFP score ranges from 0 (non compliance) to 10 points (perfect compliance), and the total SFP score ranges from 0 (lowest adherence) to 140 plus 30 extra points (highest adherence).

\section{Outcome assessment}

The anthropometric measurements of body weight, height and waist circumference (WC) were performed at recruitment using standardized procedures ${ }^{(25)}$. BMI was calculated as weight in kilograms divided by the square of height in metres. Participants were classified as non-obese $\left(\mathrm{BMI}<30 \mathrm{~kg} / \mathrm{m}^{2}\right)$ and obese $\left(\mathrm{BMI} \geq 30 \mathrm{~kg} / \mathrm{m}^{2}\right)^{(26)}$. According to WC, the participants were classified into normal and moderately increased $(<102 \mathrm{~cm}$ and $<88 \mathrm{~cm})$ and large $(\geq 102 \mathrm{~cm}$ and $\geq 88 \mathrm{~cm})$, in men and women respectively, as a proxy of abdominal obesity ${ }^{(27)}$.

\section{Assessment of other covariates}

Information on sociodemographic characteristics and lifestyle variables was also collected at recruitment. The questionnaire included information on smoking habits, highest educational level achieved, physical activity ${ }^{(28)}$ and self-reported chronic diseases ${ }^{(20,21)}$.

\section{Statistical analysis}

Quintiles of adherence to the SFP score were calculated for the whole cohort as well as for men and women separately. To describe baseline characteristics by the SFP score, continuous variables were presented as mean and standard deviation and categorical variables as percentage. Differences across the SFP score quintiles were evaluated by using the $\chi^{2}$ test for categorical variables and ANOVA for continuous variables, or Kruskal-Wallis, as appropriate.

The odds ratios of obesity defined by the BMI (normal weight and overweight $v$. obesity) and of abdominal obesity defined by the WC (normal and moderate $v$. large) were assessed through logistic regression analysis, considering the adherence to the SFP score (independent variable) categorically as quintiles. The reference category was set to the first quintile of adherence to the SFP score. The same approach was applied to evaluate the risk of overweight and obesity against normal weight (normal weight $v$. overweight and obesity) or moderate and large against normal WC (normal $v$. moderate and large). $P$ trend tests across quintiles were conducted by including the median value of each quintile as a continuous variable into the model. Risk estimates on a continuous scale were also evaluated per 10-point increase in adherence to the SFP score.

Three logistic regression models were used. The first model was adjusted for age, sex and total energy intake $(\mathrm{kJ} / \mathrm{d}$ $(\mathrm{kcal} / \mathrm{d}))$. The second model was further adjusted for smoking status, educational level and misreporting of energy intake, considering under- and over-reporters of energy intake according to the classification proposed by Mendez et $a{ }^{(29)}$. In a third model we adjusted mutually for BMI or WC, in order to assess the independent effect of the SFP score on these variables. The models were adjusted for all these variables because risk estimates changed by more than $10 \%$; other possible confounding variables (menopausal status, hypertension) did not appreciably change risk estimates and were therefore not included in the models.

In order to identify the main contributor of the SFP score to obesity risk, new scores were developed excluding one by one at each time a different component of the score.

Effect modification by sex, age ( $<50$ years, $\geq 50$ years), education level (up to secondary school $v$. higher level), physical activity (inactive and moderately inactive, active and moderately active) ${ }^{(28)}$, menopausal status (premenopausal, postmenopausal) and smoking status (current, former, never smokers) were explored by modelling interaction terms between these variables and the SPF score and by conducting stratified analysis by these variables.

Sensitivity analyses were performed by excluding participants with co-morbid conditions of obesity (hyperlipidaemia and hypertension; $n$ 2018), by excluding misreporters of energy intake ( $n$ 1864) ${ }^{(29)}$ and by using the non-energy standardized SFP score.

$P$ values were based on two-sided tests and significance was considered at the $5 \%$ level. The Stata statistical software package release $12 \cdot 0$ was used for data analysis. 


\section{Results}

Baseline characteristics of the study population by sexspecific quintiles of adherence to the SFP score are shown in Table 2 . The score ranged from 42 points (lowest adherence) to 138 points (highest adherence). On average, participants with higher adherence to the SFP consumed less alcohol, had a higher educational level, were more physically active, had a lower BMI and WC, and were less frequently smokers. Of the diet components, intake of food groups changed in the expected direction (i.e. towards higher intakes for food groups recommended at the SFP base and towards lower intakes for occasionally recommended food groups). The percentage of obese men decreased across the quintiles,

Table 2 Diet and lifestyle characteristics at baseline by sex-specific quintiles of adherence to the SFP score among 6717 participants aged 35-69 years, EPIC-Granada study

\begin{tabular}{|c|c|c|c|c|c|c|c|c|c|c|c|c|c|c|}
\hline \multirow{4}{*}{$\begin{array}{l}\text { SFP score quintile } \\
\text { Median score } \\
\text { Range }\end{array}$} & \multicolumn{6}{|c|}{ Men } & \multirow[b]{4}{*}{$P$ value* } & \multicolumn{6}{|c|}{ Women } & \multirow[b]{4}{*}{$P$ value } \\
\hline & \multicolumn{2}{|c|}{ Q1 } & \multicolumn{2}{|c|}{ Q3 } & \multicolumn{2}{|c|}{ Q5 } & & \multicolumn{2}{|c|}{ Q1 } & \multicolumn{2}{|c|}{ Q3 } & \multicolumn{2}{|c|}{ Q5 } & \\
\hline & \multicolumn{2}{|c|}{$\begin{array}{c}64 \cdot 2 \\
42 \cdot 5-62 \cdot 3\end{array}$} & \multicolumn{2}{|c|}{$\begin{array}{c}80 \cdot 9 \\
70 \cdot 4-76 \cdot 8\end{array}$} & \multicolumn{2}{|c|}{$\begin{array}{c}101 \cdot 4 \\
86 \cdot 2-129 \cdot 0\end{array}$} & & \multicolumn{2}{|c|}{$\begin{array}{c}71 \cdot 3 \\
41 \cdot 6-77 \cdot 2\end{array}$} & \multicolumn{2}{|c|}{$\begin{array}{c}89 \cdot 6 \\
85 \cdot 6-93 \cdot 7\end{array}$} & \multicolumn{2}{|c|}{$\begin{array}{c}101 \cdot 6 \\
103 \cdot 2-137 \cdot 8\end{array}$} & \\
\hline & Mean & SD & Mean & SD & Mean & SD & & Mean & SD & Mean & SD & Mean & SD & \\
\hline Age (years) & $50 \cdot 8$ & $7 \cdot 8$ & $51 \cdot 2$ & 7.9 & $51 \cdot 2$ & 7.5 & 0.232 & $48 \cdot 0$ & $8 \cdot 8$ & 48.7 & 8.5 & $49 \cdot 7$ & 8.5 & 0.591 \\
\hline Weight & 81.9 & 11.7 & $82 \cdot 2$ & 10.9 & 81.0 & 11.0 & 0.059 & $69 \cdot 3$ & $12 \cdot 0$ & $70 \cdot 8$ & 11.5 & 70.5 & $11 \cdot 1$ & 0.537 \\
\hline $\operatorname{BMI}\left(\mathrm{kg} / \mathrm{m}^{2}\right)$ & $29 \cdot 1$ & 3.7 & 28.8 & $4 \cdot 1$ & 28.7 & 3.5 & 0.071 & 28.7 & $5 \cdot 1$ & $29 \cdot 3$ & 5.0 & $29 \cdot 1$ & 4.5 & 0.052 \\
\hline WC $(\mathrm{cm})$ & $100 \cdot 8$ & $10 \cdot 0$ & $100 \cdot 3$ & $10 \cdot 0$ & $98 \cdot 6$ & 9.4 & 0.002 & $87 \cdot 6$ & $12 \cdot 3$ & 88.2 & 11.4 & $87 \cdot 3$ & $11 \cdot 1$ & 0.001 \\
\hline \multicolumn{15}{|c|}{ Dietary components $(\mathrm{g} / \mathrm{d})$ of the SFP scoret } \\
\hline Dairy products & $213 \cdot 1$ & $152 \cdot 3$ & $280 \cdot 9$ & $177 \cdot 7$ & $283 \cdot 1$ & $165 \cdot 7$ & $<0.001$ & $359 \cdot 0$ & $217 \cdot 8$ & $388 \cdot 7$ & $206 \cdot 5$ & $407 \cdot 3$ & $224 \cdot 1$ & $<0.001$ \\
\hline Rice, cereals & $55 \cdot 3$ & 30.4 & $65 \cdot 1$ & 34.9 & $80 \cdot 4$ & 41.0 & $<0.001$ & $65 \cdot 1$ & 39.9 & $69 \cdot 3$ & $36 \cdot 0$ & $72 \cdot 3$ & $36 \cdot 0$ & $<0.001$ \\
\hline Bread & 143.5 & $62 \cdot 3$ & $156 \cdot 8$ & $70 \cdot 4$ & $156 \cdot 0$ & $62 \cdot 2$ & 0.054 & $150 \cdot 7$ & 71.5 & $148 \cdot 2$ & $66 \cdot 7$ & $120 \cdot 6$ & 63.4 & $<0.001$ \\
\hline Potatoes & 53.9 & $47 \cdot 7$ & $62 \cdot 8$ & 40.47 & $66 \cdot 7$ & 39.1 & $<0.001$ & 59.5 & 39.0 & $65 \cdot 0$ & $34 \cdot 1$ & 63.0 & $37 \cdot 7$ & 0.028 \\
\hline Vegetables & $145 \cdot 9$ & 87.0 & $220 \cdot 2$ & 113.5 & 382.4 & $170 \cdot 1$ & $<0.001$ & $167 \cdot 7$ & 89.3 & 274.2 & $124 \cdot 3$ & 437.9 & $186 \cdot 2$ & $<0.001$ \\
\hline Fruits & $147 \cdot 3$ & $114 \cdot 0$ & 263.5 & 158.5 & 461.9 & 258.3 & $<0.001$ & $188 \cdot 8$ & $126 \cdot 9$ & $348 \cdot 0$ & 197.9 & $561 \cdot 1$ & $248 \cdot 7$ & $<0.001$ \\
\hline Olive oil & 13.9 & 8.3 & 21.6 & 10.4 & 29.4 & $11 \cdot 7$ & $<0.001$ & $15 \cdot 6$ & $9 \cdot 3$ & $23 \cdot 8$ & $10 \cdot 8$ & $30 \cdot 7$ & $11 \cdot 7$ & $<0.001$ \\
\hline Fish & $60 \cdot 3$ & $43 \cdot 8$ & $65 \cdot 3$ & $47 \cdot 8$ & 73.3 & $40 \cdot 2$ & $<0.001$ & $47 \cdot 7$ & 39.5 & $60 \cdot 7$ & $40 \cdot 8$ & 74.8 & $40 \cdot 2$ & $<0.001$ \\
\hline Lean meat, poultry, eggs & $50 \cdot 3$ & 41.0 & $45 \cdot 8$ & $31 \cdot 1$ & 51.0 & 23.1 & 0.002 & $60 \cdot 7$ & 46.4 & $66 \cdot 8$ & $35 \cdot 9$ & 78.2 & $40 \cdot \overline{6}$ & $<0.001$ \\
\hline Legumes & 39.5 & 28.7 & $45 \cdot 6$ & $28 \cdot 1$ & $49 \cdot 8$ & $25 \cdot 9$ & $<0.010$ & 38.5 & $30 \cdot 2$ & 44.5 & $26 \cdot 1$ & 43.8 & $26 \cdot 2$ & 0.002 \\
\hline Nuts & 9.4 & $22 \cdot 0$ & 8.8 & $12 \cdot 4$ & 8.2 & $12 \cdot 4$ & 0.001 & 8.6 & $16 \cdot 4$ & $6 \cdot 0$ & 11.0 & 6.9 & $11 \cdot 1$ & $<0.001$ \\
\hline Butter, margarine & $2 \cdot 7$ & $6 \cdot 2$ & $2 \cdot 4$ & 4.5 & $2 \cdot 0$ & 3.9 & $<0.001$ & $6 \cdot 7$ & 9.2 & 4.5 & $6 \cdot 6$ & 2.9 & 5.4 & $<0.001$ \\
\hline Fatty meats \& meat products & $76 \cdot 0$ & $44 \cdot 2$ & 60.5 & 38.9 & $41 \cdot 8$ & $34 \cdot 0$ & $<0.001$ & $64 \cdot 8$ & $42 \cdot 9$ & $50 \cdot 8$ & 33.4 & $36 \cdot 3$ & $26 \cdot 6$ & $<0.001$ \\
\hline Sweets, cakes, drinks & 124.8 & $140 \cdot 0$ & $107 \cdot 3$ & $110 \cdot 1$ & 83.0 & $100 \cdot 5$ & $<0.001$ & 169.9 & 184.4 & $110 \cdot 2$ & $124 \cdot 3$ & 90.5 & 111.4 & $<0.001$ \\
\hline Wine, beer & $369 \cdot 4$ & $251 \cdot 2$ & $164 \cdot 2$ & 189.9 & $66 \cdot 6$ & $113 \cdot 2$ & $<0.001$ & 79.5 & $150 \cdot 4$ & 31.8 & 88.3 & 14.4 & 40.0 & $<0.004$ \\
\hline Energy (kJ/d) & 10770 & 2596 & 9560 & 2279 & 8431 & 2042 & $<0.001$ & 7757 & 2286 & 7109 & 1836 & 6243 & 1640 & $<0.001$ \\
\hline Energy (kcal/d) & 2574 & $620 \cdot 4$ & 2285 & 544.8 & 2015 & 488.0 & $<0.001$ & 1854 & $546 \cdot 4$ & 1699 & 438.9 & 1492 & 391.9 & $<0.001$ \\
\hline Walking (MET/h) & $18 \cdot 9$ & $18 \cdot 2$ & 21.9 & $18 \cdot 6$ & $24 \cdot 8$ & $17 \cdot 5$ & $<0.001$ & 14.9 & $13 \cdot 5$ & $18 \cdot 3$ & $13 \cdot 0$ & $23 \cdot 3$ & $13 \cdot 8$ & $<0.001$ \\
\hline Alcohol $(g / d)$ & $33 \cdot 5$ & $25 \cdot \overline{5}$ & $12 \cdot 0$ & 14.9 & 4.7 & $8 \cdot 4$ & $<0.001$ & 3.9 & 8.0 & 1.4 & 3.9 & 0.6 & $1 \cdot 8$ & $<0.001$ \\
\hline & $n$ & $\%$ & $n$ & $\%$ & $n$ & $\%$ & $P$ value $^{*}$ & $n$ & $\%$ & $n$ & $\%$ & $n$ & $\%$ & $P$ value ${ }^{\star}$ \\
\hline BMI category & & & & & & & & & & & & & & \\
\hline $\begin{array}{l}\text { Normal weight and } \\
\text { overweight }\end{array}$ & 175 & $59 \cdot 7$ & 196 & $66 \cdot 9$ & 203 & $69 \cdot 3$ & & 674 & $64 \cdot 1$ & 632 & $60 \cdot 1$ & 654 & $62 \cdot 3$ & \\
\hline Obese & 118 & $40 \cdot 3$ & 97 & $33 \cdot 1$ & 90 & $30 \cdot 7$ & 0.009 & 377 & $35 \cdot 9$ & 419 & $39 \cdot 9$ & 396 & $37 \cdot 7$ & $0 \cdot 174$ \\
\hline WC & & & & & & & & & & & & & & \\
\hline Normal and moderate & 157 & $53 \cdot 6$ & 160 & 54.6 & 192 & 65.5 & & 558 & $53 \cdot 1$ & 517 & $49 \cdot 2$ & 564 & $53 \cdot 7$ & \\
\hline Large & 136 & $46 \cdot 4$ & 133 & 45.4 & 101 & 34.5 & 0.001 & 493 & $46 \cdot 9$ & 534 & $50 \cdot 8$ & 486 & $46 \cdot 3$ & 0.004 \\
\hline Physical activity $\ddagger$ & & & & & & & & & & & & & & \\
\hline Inactive & 75 & $25 \cdot 6$ & 75 & $25 \cdot 6$ & 83 & 28.3 & & 642 & $61 \cdot 1$ & 650 & $61 \cdot 8$ & 573 & 54.6 & \\
\hline Moderately inactive & 104 & 35.5 & 96 & $32 \cdot 7$ & 99 & 33.8 & & 327 & $31 \cdot 1$ & 286 & $27 \cdot 2$ & 319 & $30 \cdot 4$ & \\
\hline Moderately active & 56 & $19 \cdot 1$ & 67 & $22 \cdot 9$ & 56 & $19 \cdot 1$ & & 62 & 5.9 & 84 & 8.0 & 119 & $11 \cdot 3$ & \\
\hline Active & 58 & $19 \cdot 8$ & 55 & 18.8 & 55 & 18.7 & $<0.001$ & 20 & 1.9 & 31 & $3 \cdot 0$ & 39 & 3.7 & $<0.001$ \\
\hline Educational level§ & & & & & & & & & & & & & & \\
\hline Up to secondary school & 191 & $65 \cdot 2$ & 174 & $59 \cdot 4$ & 157 & 53.6 & & 843 & $80 \cdot 2$ & 857 & 81.5 & 809 & $77 \cdot 0$ & \\
\hline More than secondary school & 98 & 33.4 & 114 & 38.9 & 132 & $45 \cdot 1$ & 0.001 & 201 & $19 \cdot 1$ & 180 & $17 \cdot 1$ & 227 & $21 \cdot 6$ & 0.01 \\
\hline Smoking status & & & & & & & & & & & & & & \\
\hline Never & 67 & $22 \cdot 9$ & 87 & 29.7 & 115 & 39.2 & & 747 & $71 \cdot 1$ & 833 & $79 \cdot 3$ & 813 & 77.4 & \\
\hline Former & 110 & 37.5 & 107 & 36.5 & 106 & $36 \cdot 2$ & & 86 & $8 \cdot 2$ & 78 & 7.4 & 99 & 9.4 & \\
\hline Current smoker & 116 & $39 \cdot 6$ & 99 & 33.8 & 72 & 24.6 & $<0.001$ & 218 & $20 \cdot 7$ & 140 & $13 \cdot 3$ & 138 & $13 \cdot 1$ & $<0.001$ \\
\hline
\end{tabular}

SFP, Spanish Food Pyramid; EPIC, European Prospective Investigation into Cancer and Nutrition; WC, waist circumference; MET, metabolic equivalents.

${ }^{*} \chi^{2}$ test for categorical variables and ANOVA for continuous variables, or Kruskal-Wallis, where appropriate.

†Intake of all food groups (g/d) was standardized to $8368 \mathrm{~kJ} / \mathrm{d}(2000 \mathrm{kcal} / \mathrm{d})$.

$\ddagger$ Physical activity levels according to the Physical Activity Index ${ }^{(28)}$.

§Missing, $n 76$. 
while in women no clear trend was observed. The percentage of participants who had abdominal obesity was lower in the fifth compared with the first quintile.

Adjusted OR estimates for obesity, defined as BMI and WC, are presented in Tables 3 and 4, respectively.
A statistically significant inverse association between adherence to the SFP score and obesity was observed in men, but not in women ( $P$ value for interaction by sex $=0 \cdot 02$ ). After adjustment for potential confounders, the association remained statistically significant in men: a

Table 3 Odd ratios (95\% confidence intervals) for obesity, defined as BMI (normal weight and overweight $v$. obesity), by quintiles of adherence to the SFP score among 6717 participants aged 35-69 years, EPIC-Granada study

\begin{tabular}{|c|c|c|c|c|c|c|c|c|c|c|c|c|c|}
\hline & \multicolumn{10}{|c|}{ SFP score categories (quintiles) } & \multirow[b]{3}{*}{$P$ trend } & \multirow{2}{*}{\multicolumn{2}{|c|}{$\begin{array}{l}\text { Per 10-point increase } \\
\text { in adherence }\end{array}$}} \\
\hline & \multicolumn{2}{|r|}{ Q1 } & \multicolumn{2}{|r|}{ Q2 } & \multicolumn{2}{|r|}{ Q3 } & \multicolumn{2}{|r|}{ Q4 } & \multicolumn{2}{|r|}{ Q5 } & & & \\
\hline & OR & $95 \% \mathrm{Cl}$ & OR & $95 \% \mathrm{Cl}$ & OR & $95 \% \mathrm{Cl}$ & OR & $95 \% \mathrm{Cl}$ & OR & $95 \% \mathrm{Cl}$ & & OR & $95 \% \mathrm{Cl}$ \\
\hline \multicolumn{14}{|l|}{ Men and women } \\
\hline $\begin{array}{l}\text { SPF score } \\
\text { Normal weight and }\end{array}$ & \multirow{2}{*}{\multicolumn{2}{|c|}{$\begin{array}{c}<75.0 \\
854 / 490\end{array}$}} & \multirow{2}{*}{\multicolumn{2}{|c|}{$\begin{array}{l}75 \cdot 0-83 \cdot 8 \\
858 / 485\end{array}$}} & \multirow{2}{*}{\multicolumn{2}{|c|}{$\begin{array}{l}83 \cdot 8-91 \cdot 9 \\
825 / 519\end{array}$}} & \multirow{2}{*}{\multicolumn{2}{|c|}{$\begin{array}{c}91 \cdot 9-101 \cdot 8 \\
842 / 501\end{array}$}} & \multirow{2}{*}{\multicolumn{2}{|c|}{$\begin{array}{r}>101.8 \\
837 / 506\end{array}$}} & & & \\
\hline overweight $v$. obesity & & & & & & & & & & & & & \\
\hline Model 1 & 1 & ref. & 0.92 & $0.78,1.08$ & 0.98 & $0.83,1.15$ & 0.89 & $0.75,1.06$ & 0.82 & $0.69,0.97$ & 0.025 & 0.96 & $0.92,0.99$ \\
\hline Model 2 & 1 & ref. & 0.89 & $0.74,1.06$ & 0.92 & $0.78,1.10$ & 0.85 & $0.71,1.02$ & 0.76 & $0.63,0.91$ & 0.004 & 0.94 & $0.91,0.98$ \\
\hline Model 3 & 1 & ref. & 0.85 & $0.67,1.08$ & 0.98 & $0.77,1.23$ & $1 \cdot 14$ & $0.89,1.45$ & $1 \cdot 11$ & $0.87,1.43$ & 0.084 & 1.04 & $0.99,1.10$ \\
\hline \multicolumn{14}{|l|}{ Men } \\
\hline SPF score & \multirow{2}{*}{\multicolumn{2}{|c|}{$\begin{array}{c}<62 \cdot 3 \\
175 / 118\end{array}$}} & \multirow{2}{*}{\multicolumn{2}{|c|}{$\begin{array}{c}62 \cdot 3-70 \cdot 4 \\
184 / 109\end{array}$}} & \multirow{2}{*}{\multicolumn{2}{|c|}{$\begin{array}{c}70 \cdot 4-76 \cdot 8 \\
196 / 97\end{array}$}} & \multirow{2}{*}{\multicolumn{2}{|c|}{$\begin{array}{c}76 \cdot 8-86 \cdot 2 \\
203 / 90\end{array}$}} & \multirow{2}{*}{\multicolumn{2}{|c|}{$\begin{array}{r}>86 \cdot 2 \\
203 / 90\end{array}$}} & & & \\
\hline $\begin{array}{l}\text { Normal weight and } \\
\text { overweight } v \text {. obesity }\end{array}$ & & & & & & & & & & & & & \\
\hline Model 1 & 1 & ref. & 0.84 & $0.60,1.17$ & 0.68 & $0.48,0.96$ & 0.60 & $0.42,0.85$ & 0.57 & $0.40,0.82$ & 0.001 & 0.86 & $0.79,0.93$ \\
\hline Model 2 & 1 & ref. & 0.84 & $0.59,1.19$ & 0.68 & $0.47,0.97$ & 0.61 & $0.42,0.88$ & 0.58 & $0.40,0.87$ & 0.002 & 0.86 & $0.79,0.94$ \\
\hline Model 3 & 1 & ref. & 1.00 & $0.62,1.62$ & 0.58 & $0.35,0.96$ & 0.68 & $0.41,1.13$ & 0.85 & $0.51,1.43$ & 0.396 & 0.91 & $0.81,1.03$ \\
\hline \multicolumn{14}{|l|}{ Women } \\
\hline SPF score & \multirow{2}{*}{\multicolumn{2}{|c|}{$\begin{array}{c}<77 \cdot 2 \\
674 / 377\end{array}$}} & \multirow{2}{*}{\multicolumn{2}{|c|}{$\begin{array}{c}77 \cdot 2-85 \cdot 6 \\
672 / 378\end{array}$}} & \multirow{2}{*}{\multicolumn{2}{|c|}{$\begin{array}{c}85 \cdot 6-93 \cdot 7 \\
632 / 419\end{array}$}} & \multirow{2}{*}{\multicolumn{2}{|c|}{$\begin{array}{c}93 \cdot 7-103 \cdot 2 \\
623 / 427\end{array}$}} & \multirow{2}{*}{\multicolumn{2}{|c|}{$\begin{array}{r}>103 \cdot 2 \\
654 / 396\end{array}$}} & & & \\
\hline $\begin{array}{l}\text { Normal weight and } \\
\text { overweight } v \text { obesity }\end{array}$ & & & & & & & & & & & & & \\
\hline Model 1 & 1 & ref. & 0.98 & $0.81,1.19$ & 1.09 & $0.91,1.32$ & 1.08 & $0.89,1.30$ & 0.87 & $0.72,1.05$ & 0.316 & 0.98 & $0.94,1.02$ \\
\hline Model 2 & 1 & ref. & 0.94 & $0.77,1.14$ & 1.02 & $0.84,1.25$ & 1.01 & $0.83,1.24$ & 0.81 & $0.66,1.00$ & 0.106 & 0.96 & $0.92,1.01$ \\
\hline Model 3 & 1 & ref. & 1.01 & $0.77,1.33$ & 1.21 & $0.92,1.59$ & 1.46 & $1.11,1.92$ & 1.27 & $0.96,1.68$ & 0.061 & 1.08 & $1.00,1.15$ \\
\hline
\end{tabular}

SFP, Spanish Food Pyramid; EPIC, European Prospective Investigation into Cancer and Nutrition; ref., reference category; WC, waist circumference. Model 1: adjusted for age (years), energy intake $(\mathrm{kJ} / \mathrm{d}(\mathrm{kcal} / \mathrm{d})$ ) and sex (in models combining men and women).

Model 2: further adjusted for smoking status (never, former, current smoker), educational level (none, primary, secondary, professional school, university degree, missing), misreporting of energy intake (under-reporters, acceptable reporters and over-reporters) and sex (in models combining men and women).

Model 3: further adjusted for WC (cm).

Table 4 Odd ratios (95\% confidence intervals) for abdominal obesity, defined as WC (normal and moderate $v$. large), by quintiles of adherence to the SFP score among 6717 participants aged 35-69 years, EPIC-Granada study

\begin{tabular}{|c|c|c|c|c|c|c|c|c|c|c|c|c|c|}
\hline & \multicolumn{10}{|c|}{ SFP score categories (quintiles) } & \multirow[b]{3}{*}{$P$ trend } & \multirow{2}{*}{\multicolumn{2}{|c|}{$\begin{array}{l}\text { Per 10-point increase } \\
\text { in adherence }\end{array}$}} \\
\hline & \multicolumn{2}{|r|}{ Q1 } & \multicolumn{2}{|r|}{ Q2 } & \multicolumn{2}{|r|}{ Q3 } & \multicolumn{2}{|r|}{ Q4 } & \multicolumn{2}{|r|}{ Q5 } & & & \\
\hline & OR & $95 \% \mathrm{Cl}$ & OR & $95 \% \mathrm{Cl}$ & OR & $95 \% \mathrm{Cl}$ & OR & $95 \% \mathrm{Cl}$ & OR & $95 \% \mathrm{Cl}$ & & OR & $95 \% \mathrm{Cl}$ \\
\hline \multicolumn{14}{|l|}{ Men and women } \\
\hline SPF score & \multirow{2}{*}{\multicolumn{2}{|c|}{$\begin{array}{c}<75.0 \\
740 / 604\end{array}$}} & \multirow{2}{*}{\multicolumn{2}{|c|}{$\begin{array}{c}75 \cdot 0-83 \cdot 8 \\
704 / 639\end{array}$}} & \multirow{2}{*}{\multicolumn{2}{|c|}{$\begin{array}{c}83 \cdot 8-91 \cdot 9 \\
682 / 662\end{array}$}} & \multirow{2}{*}{\multicolumn{2}{|c|}{$\begin{array}{c}91.9-101 \cdot 8 \\
729 / 614\end{array}$}} & \multirow{2}{*}{\multicolumn{2}{|c|}{$\begin{array}{r}>101 \cdot 8 \\
731 / 612\end{array}$}} & & & \\
\hline Normal and moderate $v$. large & & & & & & & & & & & & & \\
\hline Model 1 & 1 & ref. & 1.04 & $0.88,1.22$ & 1.04 & $0.88,1.22$ & 0.86 & $0.72,1.01$ & 0.75 & $0.63,0.89$ & $<0.001$ & 0.93 & $0.90,0.96$ \\
\hline Model 2 & 1 & ref. & 1.02 & $0.85,1.21$ & 0.99 & $0.83,1.18$ & 0.82 & $0.69,0.98$ & 0.69 & $0.57,0.83$ & $<0.001$ & 0.91 & $0.88,0.95$ \\
\hline Model 3 & 1 & ref. & 1.07 & $0.84,1.35$ & 0.97 & $0.76,1.24$ & 0.80 & $0.63,1.02$ & 0.63 & $0.49,0.81$ & $<0.001$ & 0.88 & $0.84,0.93$ \\
\hline \multicolumn{14}{|l|}{ Men } \\
\hline SPF score & \multirow{2}{*}{\multicolumn{2}{|c|}{$\begin{array}{c}<62 \cdot 3 \\
157 / 136\end{array}$}} & \multirow{2}{*}{\multicolumn{2}{|c|}{$\begin{array}{c}62 \cdot 3-70 \cdot 4 \\
173 / 120\end{array}$}} & \multirow{2}{*}{\multicolumn{2}{|c|}{$\begin{array}{c}70 \cdot 4-76 \cdot 8 \\
160 / 133\end{array}$}} & \multirow{2}{*}{\multicolumn{2}{|c|}{$\begin{array}{c}76 \cdot 8-86 \cdot 2 \\
181 / 112\end{array}$}} & \multirow{2}{*}{\multicolumn{2}{|c|}{$\begin{array}{c}>86 \cdot 2 \\
192 / 101\end{array}$}} & & & \\
\hline Normal and moderate $v$. large & & & & & & & & & & & & & \\
\hline Model 1 & 1 & ref. & 0.76 & $0.54,1.07$ & 0.89 & $0.64,1.25$ & 0.65 & $0.46,0.92$ & 0.53 & $0.37,0.76$ & $<0.001$ & 0.86 & $0.80,0.93$ \\
\hline Model 2 & 1 & ref. & 0.77 & $0.54,1.09$ & 0.92 & $0.66,1.31$ & 0.68 & $0.47,0.97$ & 0.54 & $0.37,0.78$ & 0.001 & $0 \cdot 86$ & $0.80,0.94$ \\
\hline Model 3 & 1 & ref. & 0.83 & $0.50,1.38$ & $1 \cdot 14$ & $0.80,1.55$ & 0.81 & $0.49,1.35$ & 0.50 & $0.29,0.84$ & 0.006 & 0.86 & $0.76,0.96$ \\
\hline \multicolumn{14}{|l|}{ Women } \\
\hline $\begin{array}{l}\text { SPF score } \\
\text { Normal and moderate } v \text {. large }\end{array}$ & \multicolumn{2}{|c|}{$\begin{array}{c}<77 \cdot 2 \\
558 / 493\end{array}$} & \multicolumn{2}{|c|}{$\begin{array}{c}77 \cdot 2-85 \cdot 6 \\
551 / 499\end{array}$} & \multicolumn{2}{|c|}{$\begin{array}{c}85 \cdot 6-93 \cdot 7 \\
517 / 534\end{array}$} & \multicolumn{2}{|c|}{$\begin{array}{c}93 \cdot 7-103 \cdot 2 \\
533 / 517\end{array}$} & \multicolumn{2}{|c|}{$\begin{array}{r}>103 \cdot 2 \\
564 / 486\end{array}$} & & & \\
\hline Model 1 & 1 & ref. & 1.01 & $0.84,1.22$ & 1.08 & $0.89,1.30$ & 0.95 & $0.78,1.15$ & 0.76 & $0.62,0.92$ & 0.007 & 0.94 & $0.91,0.98$ \\
\hline Model 2 & 1 & ref. & 0.96 & $0.78,1.17$ & $1 \cdot 10$ & $0 \cdot 82,1 \cdot 22$ & 0.88 & $0.71,1.07$ & 0.70 & $0.57,0.86$ & $<0.001$ & 0.92 & $0.87,0.97$ \\
\hline Model 3 & 1 & ref. & 0.93 & $0 \cdot 70,1 \cdot 22$ & 0.90 & $0 \cdot 69,1 \cdot 19$ & 0.78 & $0.59,1.03$ & 0.63 & $0.47,0.83$ & 0.001 & 0.90 & $0.84,0.95$ \\
\hline
\end{tabular}

SFP, Spanish Food Pyramid; WC, waist circumference; EPIC, European Prospective Investigation into Cancer and Nutrition; ref., reference category. Model 1: adjusted for age (years), energy intake $(\mathrm{kJ} / \mathrm{d}(\mathrm{kcal} / \mathrm{d}))$ and sex (in models combining men and women).

Model 2: further adjusted for smoking status (never, former, current smoker), educational level (none, primary, secondary, professional school, university degree, missing), misreporting of energy intake (under-reporters, acceptable reporters and over-reporters) and sex (in models combining men and women).

Model 3: further adjusted for BMI $\left(\mathrm{kg} / \mathrm{m}^{2}\right)$. 
10-point increase in adherence to the SFP score was associated with a $14 \%(\mathrm{OR}=0.86 ; 95 \% \mathrm{CI} 0.79,0.94)$ lower likelihood of obesity $\left(\mathrm{OR}_{\mathrm{Q} 5} v \cdot \mathrm{Q} 1=0.58 ; 95 \% \mathrm{CI}\right.$ $0.40,0.87 ; P$ trend=0.002). Further adjustment for WC attenuated these estimates in men $\left(\mathrm{OR}_{\text {per }} 10\right.$-point increase $=$ $0.91 ; 95 \%$ CI $0.81,1.03)$ and turned the association positive in women $\left(\mathrm{OR}_{\text {per }}\right.$ 10-point increase $=1.08 ; 95 \%$ CI $1 \cdot 00,1 \cdot 15)$.

With regard to abdominal obesity (Table 4), the likelihood of being obese decreased by $9 \%(\mathrm{OR}=0.91$; $95 \%$ CI $0 \cdot 88,0.95)$ per 10-point increase in adherence to the SFP score $\left(\mathrm{OR}_{\mathrm{Q} 5} v \cdot \mathrm{Q} 1=0.69 ; 95 \%\right.$ CI $0.57,0.83$; $P$ trend $<0 \cdot 001)$. Despite no evidence for interaction by $\operatorname{sex}(P=0 \cdot 28)$, the association was stronger in men $(14 \%$ decrease, $\mathrm{OR}_{\text {per } 10 \text {-point increase }}=0.86 ; 95 \%$ CI $\left.0 \cdot 80,0.94\right)$ than in women $\left(8 \%\right.$ decrease, $\mathrm{OR}_{\text {per 10-point increase }}=0.92$; $95 \%$ CI $0.87,0.97)$. Further adjustment for BMI did not substantially change the estimates $\left(\mathrm{OR}_{\text {per } 10 \text {-point increase }}=\right.$ $0 \cdot 88 ; 95 \%$ CI 0.84, 0.93), although the association weakened slightly in men $\left(\mathrm{OR}_{\text {per }}{ }_{10 \text {-point }}\right.$ increase $=0.86$; $95 \%$ CI $0.76,0.96)$ and strengthened in women $\left(\mathrm{OR}_{\text {per 10-point increase }}=0.90 ; 95 \%\right.$ CI 0.84, 0.95).

No significant association was observed between adherence to the SFP score and risk of becoming overweight and obese compared with normal weight or moderately and largely abdominally obese compared with normal WC (results not shown).

Interaction analyses revealed statistically significant interactions only between the SFP score and physical activity for BMI $(P=0.01)$ and a borderline interaction for WC $(P=0.08)$. In the fully adjusted models, the effect of higher adherence to the SFP score on obesity was stronger and statistically significant in physically active men $\left(\mathrm{OR}_{\text {per 10-point increase }}=0 \cdot 80 ; 95 \% \mathrm{CI} 0 \cdot 65,0.99\right)$, but not so in those who were inactive $(P$ for interaction $=0.03)$.
Interestingly, the association with obesity was largely attenuated in physically active women $\left(\mathrm{OR}_{\text {per }}\right.$ 10-point increase $=$ $1.00 ; 95 \%$ CI $0.81,1.24)$. The effect of the SFP score on abdominal obesity was more marked in physically inactive men $\left(\mathrm{OR}_{\text {per }}\right.$ 10-point increase $\left.=0.79 ; 95 \% \mathrm{CI} 0.68,0.92\right)$ and women $\left(\mathrm{OR}_{\text {per 10-point increase }}=0.89 ; 95 \%\right.$ CI 0.84, 0.95) compared with physically active participants $\left(\mathrm{OR}_{\text {per }}\right.$ 10-point increase $=$ 0.95; $95 \%$ CI 0.83, 1.09; see online supplementary material, Supplemental Table 1).

Table 5 shows risk estimates for each component of the SFP score. The subtraction of the physical activity recommendation (walking for $30 \mathrm{~min} / \mathrm{d}$ ) from the SFP score resulted in a higher likelihood of being obese $(\mathrm{OR}=0.96$; $95 \%$ CI 0.92, 0.99 for BMI and OR=0.93; $95 \%$ CI 0.89, 0.99 for WC), although the association remained inverse and statistically significant.

The sensitivity analyses results are shown in Table 6. Excluding participants with self-reported co-morbid conditions showed no substantial effect on the estimates. However, estimates were considerably affected after excluding misreporters of energy intake: the association between adherence to the SPF score and obesity was weakened in men, but got stronger in women, reaching statistical significance for obesity $\left(\mathrm{OR}_{\text {per } 10-\text { point increase }}=0.93 ; 95 \% \mathrm{CI}\right.$ $0 \cdot 88,0.97)$. The associations were only slightly weakened when using the non-energy standardized SPF score.

\section{Discussion}

A higher adherence to the current Spanish dietary guidelines ${ }^{(7)}$ was significantly associated with a lower odds of being abdominally obese in both men and women, and with lower odds of obesity as defined by BMI in men only. Specifically, a 10-point increase in adherence to the SFP

Table 5 Odds ratios (95\% confidence intervals) per 10-point increase in adherence to the SFP score for obesity defined as BMI and WC, after subtraction of each dietary component from the SFP score, among 6717 participants aged 35-69 years, EPIC-Granada study

\begin{tabular}{|c|c|c|c|c|c|c|}
\hline \multirow[b]{2}{*}{ Components of the SFP score } & \multicolumn{2}{|c|}{$\mathrm{BMI}^{*}$} & \multirow[b]{2}{*}{$P$ value } & \multicolumn{2}{|c|}{$W C^{*}$} & \multirow[b]{2}{*}{$P$ value } \\
\hline & OR† & $95 \% \mathrm{Cl}$ & & OR† & $95 \% \mathrm{Cl}$ & \\
\hline SFP score & 0.94 & $0.91,0.98$ & 0.002 & 0.91 & $0.88,0.95$ & $<0.001$ \\
\hline SFP score minus potatoes, rice and bread & 0.94 & $0.90,0.97$ & 0.001 & 0.90 & $0.87,0.94$ & $<0.001$ \\
\hline SFP score minus vegetables & 0.92 & $0.88,0.96$ & $<0.001$ & 0.91 & $0.87,0.94$ & $<0.001$ \\
\hline SFP score minus fruits & 0.93 & $0.89,0.98$ & 0.004 & 0.91 & $0.87,0.95$ & $<0.001$ \\
\hline SFP score minus olive oil & 0.94 & $0.90,0.98$ & 0.002 & 0.91 & $0.87,0.94$ & $<0.001$ \\
\hline SFP score minus dairy products & 0.95 & $0.92,0.98$ & 0.001 & 0.92 & $0.89,0.96$ & 0.001 \\
\hline SFP score minus fish & 0.94 & $0.90,0.98$ & 0.002 & 0.90 & $0.87,0.94$ & $<0.001$ \\
\hline SFP score minus lean meat, poultry, eggs & 0.94 & $0.90,0.97$ & 0.001 & 0.90 & $0.87,0.94$ & $<0.001$ \\
\hline SFP score minus legumes & 0.94 & $0.90,0.98$ & 0.003 & 0.91 & $0.87,0.95$ & $<0.001$ \\
\hline SFP score minus nuts & 0.95 & $0.91,0.98$ & 0.006 & 0.91 & $0.88,0.95$ & $<0.001$ \\
\hline SFP score minus fatty meat and meat products & 0.95 & $0.91,0.99$ & 0.007 & 0.91 & $0.88,0.95$ & $<0.001$ \\
\hline SFP score minus sweets, snacks, cakes & 0.94 & $0.90,0.98$ & 0.002 & 0.89 & $0.85,0.93$ & $<0.001$ \\
\hline SFP score minus butter and margarine & 0.94 & $0.90,0.98$ & 0.002 & 0.89 & $0.86,0.93$ & $<0.001$ \\
\hline SFP score minus alcohol (wine \& beer) & 0.93 & $0.90,0.97$ & 0.001 & 0.90 & $0.86,0.94$ & $<0.001$ \\
\hline SFP score minus physical activity (walking) & 0.96 & $0.92,0.99$ & 0.025 & 0.93 & $0.89,0.99$ & 0.014 \\
\hline
\end{tabular}

SFP, Spanish Food Pyramid; WC, waist circumference; EPIC, European Prospective Investigation into Cancer and Nutrition.

${ }^{*}$ Risk estimates are presented for men and women combined as the main contributors to obesity (walking, sweets and snacks, butter and margarine) were the same in both sexes.

†Adjusted for age (years), energy intake (kJ/d (kcal/d)), smoking status (never, former, current smoker), educational level (none, primary, secondary, professional school, university degree, missing), misreporting of energy intake (under-reporter, acceptable reporters and over-reporters) and sex. 
Table 6 Odds ratios (95\% confidence intervals) per 10-point increase in adherence to the SFP score for obesity risk defined as BMI and WC, after conducting sensitivity analyses, among 6717 participants aged 35-69 years, EPIC-Granada study

\begin{tabular}{|c|c|c|c|c|c|c|c|c|c|c|c|c|}
\hline \multirow[b]{2}{*}{ Sensitivity analysis } & \multicolumn{2}{|r|}{ BMI } & \multirow[b]{2}{*}{$P$ value } & \multicolumn{2}{|r|}{ WC } & \multirow[b]{2}{*}{$P$ value } & \multicolumn{2}{|r|}{ BMI } & \multirow[b]{2}{*}{$P$ value } & \multicolumn{2}{|c|}{ WC } & \multirow[b]{2}{*}{$P$ value } \\
\hline & $\mathrm{OR}^{*}$ & $95 \% \mathrm{Cl}$ & & $\mathrm{OR}^{*}$ & $95 \% \mathrm{Cl}$ & & OR† & $95 \% \mathrm{Cl}$ & & OR† & $95 \% \mathrm{Cl}$ & \\
\hline \multicolumn{13}{|c|}{ SFP score (models for reference) } \\
\hline All & 0.94 & $0.91,0.98$ & 0.002 & 0.91 & $0.88,0.95$ & $<0.001$ & 1.04 & $0.99,1 \cdot 10$ & 0.110 & 0.88 & $0.84,0.93$ & $<0.001$ \\
\hline Men & 0.86 & $0.79,0.94$ & 0.001 & 0.86 & $0.80,0.94$ & 0.001 & 0.91 & $0.81,1.03$ & 0.141 & 0.86 & $0.76,0.96$ & 0.010 \\
\hline Women & 0.96 & $0.92,1.01$ & 0.104 & 0.92 & $0.87,0.97$ & $<0.001$ & 1.08 & $1.00,1.15$ & 0.046 & 0.90 & $0.84,0.95$ & $<0.001$ \\
\hline \multicolumn{13}{|c|}{ Excluding participants with self-reported status of hyperlipidaemia and hypertension } \\
\hline All & 0.93 & $0.88,0.97$ & 0.002 & 0.91 & $0.87,0.95$ & $<0.001$ & 1.03 & $0.96,1.10$ & 0.415 & 0.89 & $0.83,0.95$ & $<0.001$ \\
\hline Men & 0.87 & $0.78,0.97$ & 0.014 & 0.89 & $0.80,0.98$ & 0.031 & 0.89 & $0.76,1.04$ & 0.134 & 0.89 & $0.76,1.00$ & 0.046 \\
\hline Women & 0.94 & $0.89,1.00$ & 0.064 & 0.91 & $0.87,0.96$ & 0.001 & 1.06 & $0.99,1.14$ & 0.107 & 0.89 & $0.83,0.96$ & 0.002 \\
\hline \multicolumn{13}{|c|}{ Excluding under- and over-reporters of energy intakeł } \\
\hline All & 0.92 & $0.87,0.96$ & $<0.001$ & 0.91 & $0.87,0.95$ & $<0.001$ & 1.01 & $0.95,1.07$ & 0.742 & 0.89 & $0.84,0.95$ & $<0.001$ \\
\hline Men & 0.87 & $0.79,0.96$ & 0.007 & 0.89 & $0.81,0.98$ & 0.019 & 0.96 & $0.84,1.09$ & 0.557 & 0.89 & $0.77,1.00$ & 0.046 \\
\hline Women & 0.93 & $0.88,0.97$ & 0.004 & 0.91 & $0.87,0.96$ & $<0.001$ & 1.02 & $0.96,1.09$ & 0.491 & 0.89 & $0.83,0.96$ & 0.002 \\
\hline \multicolumn{13}{|c|}{ Without energy-standardized intakes§ } \\
\hline All & 0.95 & $0.91,0.98$ & 0.008 & 0.93 & $0.89,0.97$ & 0.001 & 1.02 & $0.96,1.08$ & 0.544 & 0.91 & $0.86,0.96$ & 0.001 \\
\hline Men & 0.88 & $0.82,0.96$ & 0.003 & 0.89 & $0.83,0.98$ & 0.011 & 0.91 & $0.81,1.02$ & 0.093 & 0.90 & $0.81,0.99$ & 0.021 \\
\hline Women & 0.97 & $0.92,1.01$ & 0.169 & 0.94 & $0.89,0.98$ & 0.009 & 1.06 & $0.99,1.13$ & 0.072 & 0.91 & $0.85,0.97$ & 0.004 \\
\hline
\end{tabular}

SFP, Spanish Food Pyramid; WC, waist circumference; EPIC, European Prospective Investigation into Cancer and Nutrition.

${ }^{*}$ Adjusted for smoking status (never, former, current smoker), educational level (none, primary, secondary, professional school and university degree, missing), misreporting of energy intake (under-reporters, acceptable reporters and over-reporters) and sex (in models combining men and women).

†Further adjusted for BMI $\left(\mathrm{kg} / \mathrm{m}^{2}\right)$ or WC $(\mathrm{cm})$.

¥Without adjustment for misreporting of energy intake.

§SPF score without energy standardization (8368 kJ (2000 kcal)).

score was associated with a $12 \%$ less likelihood of abdominal obesity.

Few studies have examined the relationship between dietary scores, developed on the basis of dietary guidelines, and the risk of obesity. Most of them have focused on the Dietary Guidelines for Americans (DGA), specifically the Healthy Eating Index (HEI-05 and HEI-90). In the study conducted within the Multi-Ethnic Study of Atherosclerosis, which included 6814 men and women (aged $45-84$ years), the association between the HEI-05 and changes in BMI and WC was examined over 18 months of follow-up ${ }^{(14)}$. Both HEI indices were associated with a significant reduction in BMI and WC. Within the CARDIA (Coronary Artery Risk Development in Young Adults) study, 4913 young adults (aged 18-30 years) were followed for 20 years to examine whether diet compliance with the 2005 DGA, as measured by a revised DQI, was associated with weight gain and obesity risk. A 10-point increase in the adherence to these guidelines was associated with a $10 \%$ lower risk of gaining $10 \mathrm{~kg}$ in weight ${ }^{(16)}$. A lower HEI score was also associated with higher obesity risk $(\mathrm{OR}=1.8 ; 95 \% \mathrm{CI} 1.4,2.5)$ in a cross-sectional study that included 10930 participants (aged 20-75 years) ${ }^{(15)}$. In Europe, the French (PNNS) and the Finnish dietary guidelines (FDS) have been evaluated in relation to obesity risk. A one unit increase in adherence to the PNNS score was associated with an $11 \%$ lower incidence of obesity (95\% CI 0.80, 0.99) after a 6-year follow-up of 3531 participants (aged 54 years and older) of the SU.VI.MAX study ${ }^{(17)}$. The same study population was used to re-evaluate this and other dietary indices after 13 years of follow-up ${ }^{(18)}$, confirming the inverse association. Among the 1720 participants of the FINRISK study, those who were men and in the highest $v$. lowest FDS quintile were $36 \%(\mathrm{OR}=0.64 ; 95 \% \mathrm{CI} 0 \cdot 47,0.88)$ less likely to have abdominal obesity ${ }^{(19)}$.

Despite differences in the methods used to measure dietary intake, the scoring systems applied, the study population's age range and the study designs (crosssectional and prospective studies), these findings are generally comparable to our study. However, the comparison with these studies has to be taken cautiously due to the reasons mentioned above. Moreover, underreporting of energy intake was not accounted for and may have affected the associations reported in these studies. In our study, controlling for misreporting of energy intake strengthened the estimates. For instance, the association became stronger in women after restricting the analysis to acceptable reporters of energy intake, possibly driven by the higher proportion of under-reporters of energy intake in women $(20 \%)$. It has been reported that obese participants and women are more likely to underreport their energy intakes ${ }^{(29)}$. These participants may score therefore higher, being obese at the same time.

None of the previous studies evaluated the independent effect of the score on BMI and WC by mutually controlling for each other, which gives insight into the effect of diet quality on body fatness, i.e. overall adiposity or abdominal obesity ${ }^{(30)}$. In our study, the effect of adherence to the SFP score on the occurrence of abdominal obesity was independent of BMI. The differences found between men and women might be due to differences in body fat distribution $^{(31)}$. As such, women at menopausal ages experience changes in body fat distribution that seem to be confined to abdominal fat deposition ${ }^{(32)}$. Menopausal 
status, however, did not modify the associations in our study. It should be also taken into consideration that since the proportion of obese men was lower compared with women (1:4), statistical power to detect associations in men may have been limited.

Even though the Spanish dietary guidelines have not been evaluated yet in relation to the prevention of obesity, other indices based on the assessment of the Mediterranean dietary pattern (MD) have been explored ${ }^{(33-35)}$. Two previous studies conducted within the EPIC-Spain study focused on the effect that adherence to the MD has on weight gain ${ }^{(33)}$ and obesity risk $^{(34)}$. The association between adherence to the MD and weight change was found to be rather weak ${ }^{(33)}$, while for obesity an almost $30 \%$ reduction in risk was reported for high adherence to the $\mathrm{MD}^{(34)}$. On the contrary, in the SUN cohort (Spanish Seguimiento Universidad de Navarra study) no significant association was observed between the MD and obesity risk, although weight gain was significantly reduced after 4 years of follow-up ${ }^{(35)}$. Even though the MD pattern defined in these studies agrees with the traditional Spanish dietary patterns, there are differences in the current Spanish dietary guidelines in relation to recommendations for specific food groups, amount and frequency of food consumption, and also for practising daily physical activity. The evaluation of the Spanish dietary guidelines therefore deserves a separate investigation.

Potential mechanisms by which adhering to the Spanish dietary guidelines may protect against obesity development might be through the low energy density provided by a diet relying on these guidelines, the high fibre content and the physical activity recommendations, which are all well-established determinants of healthy weight ${ }^{(2)}$. The current study showed that the effect of a higher adherence to the SFP score on WC was stronger in physically inactive men and women, suggesting that the dietary guidelines may contribute to energy balance and, as a result, to a healthier body weight and WC in this group. The walking recommendation was the main component of the SPF score contributing to the prevention of obesity. Physical activity is indeed a very strong predictor of energy balance $^{(36)}$.

Given the limitations imposed by the cross-sectional design, it cannot be concluded whether the adherence to these guidelines leads to a lower obesity risk or whether health-conscious obese participants have recently modified their dietary habits to lose weight, thus adhering better to these guidelines. Other limitations of the present study are related to the computation of the SFP score, which was based on a subjective interpretation of the SFP. For instance, the frequency of occasional consumption of certain food groups was defined as less than twice weekly. Some adaptations were also implemented for physical activity and alcohol consumption recommendations, as for example the use of MET walking/d for the daily $30 \mathrm{~min}$ walking recommendation. It is also possible that different interpretations of the SFP may lead to different results, either null or significant associations. For instance, occasional frequency of consumption considered as more than three times weekly for sweets and snacks resulted in non-statistically significant associations. Misclassification of food groups might be also possible. Food groups were chosen from the general EPIC classification, which included seventeen main food groups and their subgroups ${ }^{(37)}$. Their categorization into the food groups defined in the SFP might be subjected to decisions made on the basis of our criterion or EPIC food groups. For instance, fruits and vegetable juices were not included in the fruits and vegetables groups because they differ nutritionally (e.g. added sugars and vitamins) and were quantified in liquid form. Commercial juices, concentrates and nectars (i.e. soft drinks, juices with added sugars) were therefore all categorized in the 'Sugary soft drinks group'. Details of food items included in fruit and vegetable subgroups have been reported elsewhere ${ }^{(38)}$. Finally, as in every dietdisease relationship study, dietary measurement error and reporting bias cannot be ruled out. Reverse causation was tested by excluding participants with self-reported comorbid conditions (hyperlipidaemia and hypertension), because they are prone to modify their dietary habits. However, estimates did not change appreciably.

Strengths of the present study include its large sample size, the use of measured anthropometric variables and a validated dietary questionnaire, and the consideration of misreporting of energy intake. The strength of the scoring method that we applied is that it was calculated proportionally to the frequency of consumption and portion size, and also accounting for the minimum and maximum recommended food intake. By using this scoring system, we considered the fact that consumption of food groups in an excessive (positive energy balance) or deficient (less benefit from nutrient-rich food) manner is unfavourable. This method is based on the one developed by von Ruesten et $a l^{(13)}$ for the German dietary guidelines, instead of using cut points for ranking individuals, as has been done in previous studies ${ }^{(14-19)}$. We standardized the SFP score for energy intake to make isoenergetic comparisons and because this procedure does not affect measures of diet quality, as was confirmed when examining the non-energy adjusted SFP score and its association with obesity.

\section{Conclusion}

The present study is the first one evaluating the Spanish dietary guidelines (SENC-2004) in relation to obesity. Our findings support that the Spanish guidelines might be an effective tool for the prevention of obesity, and should be therefore implemented in forthcoming nutritional intervention programmes targeted to the population, raising awareness about their existence and importance to follow 
them. Besides, more studies assessing the effect of the SFP on long-term weight gain and obesity risk are needed to replicate our findings and to confirm this possible causal relationship. The SFP score developed in the current study could be used further to investigate its relationship with other health and disease determinants.

\section{Acknowledgements}

Financial support: This research received no specific grant from any funding agency in the public, commercial or not-for-profit sectors. The EPIC study was supported by the European Commission (DG-SANCO); the International Agency for Research on Cancer; the Spanish Ministry of Health (ISCIII RETICC RD06/0020); Spanish Regional Governments of Andalusia, Asturias, Basque Country, Murcia and Navarra; and the Catalan Institute of Oncology. Conflict of interest: None. Authorship: E.M.-M. and I.U. carried out the data analyses and drafted the manuscript. E.M.-P. participated in the data analysis. E.M.-M., I.U. and M.-J.S. conceived of the study, and M.-J.S. participated in its coordination and helped to draft the manuscript. All authors conducted research and data collection. They also read and approved the final manuscript. Ethics statement: Approval for the EPIC-Spain study was obtained from the Medical Ethical Committee of Bellvitge Hospital.

\section{Supplementary material}

To view supplementary material for this article, please visit http://dx.doi.org/10.1017/S1368980014000688

\section{References}

1. World Health Organization (2011) Global Status Report on Non-Communicable Diseases 2010. Geneva: WHO.

2. World Health Organization (2011) Obesity and Overweight. Geneva: WHO.

3. Gutiérrez-Fisac JL, Guallar-Castillón P, León-Muñoz LM et al. (2012) Prevalence of general and abdominal obesity in the adult population of Spain, 2008-2010: the ENRICA study. Obes Rev 13, 388-392.

4. Food and Agriculture Organization of the United Nations/ World Health Organization (1998) Preparation and Use of Food-Based Dietary Guidelines. Report of a Joint FAO/WHO Consultation. WHO Technical Report Series no. 880. Geneva: WHO.

5. Aranceta J (1995) Objetivos nutricionales para la población española. Propuesta de la SENC para la población española. In Documento de Consenso Guías Alimentarias para la Población Española, pp. 127-162 [L Serra, J Aranceta and J Mataix et al., editors]. Barcelona: SGEditores.

6. Serra-Majem L, Aranceta J \& SENC Working Group (2001) Nutritional objectives for the Spanish population. Consensus from the Spanish Society of Community Nutrition. Public Health Nutr 4, 1409-1413.
7. Serra-Majem L, Aranceta J \& SENC Working Group (2004) Guía de la Alimentación Saludable. Madrid: SENC.

8. Aranceta J, Serra-Majem L \& SENC Working Group (2011) Objetivos nutricionales para la poblacion española. Rev Esp Nutr Comunitaria 17, 178-199.

9. Kourlaba G \& Panagiotakos DB (2009) Dietary quality indices and human health: a review. Maturitas $\mathbf{6 2}, 1-8$.

10. Hu FB, Rimm EB, Stampfer MJ et al. (2000) Prospective study of major dietary patterns and risk of coronary heart disease in men. Am J Clin Nutr 72, 912-921.

11. McCullough ML, Feskanich D, Stampfer MJ et al. (2002) Diet quality and major chronic disease risk in men and women: moving toward improved dietary guidance. Am J Clin Nutr 76, 1261-1271.

12. Kennedy ET, Ohls J, Carlson S et al. (1995) The Healthy Eating Index: design and applications. J Am Diet Assoc 95, 1103-1108.

13. von Ruesten A, Illner AK, Buijsse B et al. (2010) Adherence to recommendations of the German food pyramid and risk of chronic diseases: results from the EPIC-Potsdam study. Eur J Clin Nutr 64, 1251-1259.

14. Gao SK, Beresford SA, Frank LL et al. (2008) Modifications to the Healthy Eating Index and its ability to predict obesity: the Multi-Ethnic Study of Atherosclerosis. Am J Clin Nutr 88, 64-69.

15. Guo X, Warden BA, Paeratakul S et al. (2004) Healthy eating index and obesity. Eur J Clin Nutr 58, 1580-1586.

16. Zamora D, Gordon-Larsen P, Jacobs DR Jr et al. (2010) Diet quality and weight gain among black and white young adults: the Coronary Artery Risk Development in Young Adults (CARDIA) Study (1985-2005). Am J Clin Nutr 92, 784-793.

17. Kesse-Guyot E, Castetbon K, Estaquio C et al. (2009) Association between the French nutritional guideline-based score and 6-year anthropometric changes in a French middle-aged adult cohort. Am J Epidemiol 170, 757-765.

18. Lassale C, Fezeu L, Andreeva VA et al. (2012) Association between dietary scores and 13-year weight change and obesity risk in a French prospective cohort. Int $J$ Obes (Lond) 36, 1455-1462.

19. Kanerva N, Kaartinen NE, Ovaskainen ML et al. (2013) A diet following Finnish nutrition recommendations does not contribute to the current epidemic of obesity. Public Health Nutr 16, 786-794.

20. Riboli E, Hunt KJ, Slimani N et al. (2002) European Prospective Investigation into Cancer and Nutrition (EPIC): study populations and data collection. Public Health Nutr 5, 1113-1124.

21. González CA, Navarro C, Martínez C et al. (2004) El Estudio Prospectivo Europeo sobre Cáncer y Nutrición (EPIC). Rev Esp Salud Publica 78, 167-176.

22. EPIC Group of Spain (1997) Validity and reproducibility of a diet history questionnaire in Spain. (I. Foods). Int J Epidemiol 26, Suppl. 1, S91-S99.

23. EPIC Group of Spain (1997) Validity and reproducibility of a diet history questionnaire in Spain. (II. Nutrients). Int J Epidemiol 26, Suppl. 2, S100-S109.

24. Slimani N, Deharveng G, Unwin I et al. (2007) The EPIC nutrient database project (ENDB): a first attempt to standardize nutrient databases across the 10 European countries participating in the EPIC study. Eur J Clin Nutr 61, 1037-1056.

25. Haftenberger M, Lahmann PH, Panico S et al. (2002) Overweight, obesity and fat distribution in 50- to 64-yearold participants in the European Prospective Investigation into Cancer and Nutrition (EPIC). Public Health Nutr 5, 1147-1162.

26. World Health Organization (2000) Obesity: Preventing and Managing the Global Epidemic. Report of a WHO Consultation. WHO Technical Report Series. no. 894. Geneva: WHO.

27. National Cholesterol Education Program (NCEP) Expert Panel on Detection, Evaluation, and Treatment of High Blood 
Cholesterol in Adults (Adult Treatment Panel III) (2002) Third Report of the National Cholesterol Education Program (NCEP) Expert Panel on Detection, Evaluation, and Treatment of High Blood Cholesterol in Adults (Adult Treatment Panel III) final report. Circulation 106, 3143-3421.

28. Wareham NJ, Jakes RW, Rennie KL et al. (2003) Validity and repeatability of a simple index derived from the short physical activity questionnaire used in the European Prospective Investigation into Cancer and Nutrition (EPIC) study. Public Health Nutr 6, 407-413.

29. Mendez MA, Popkin BM, Buckland G et al. (2011) Alternative methods of accounting for underreporting and overreporting when measuring dietary intake-obesity relations. Am J Epidemiol 173, 448-458.

30. Hu FB (2008) Obesity Epidemiology, pp. 62-76. New York: Oxford University Press.

31. Blaak E (2001) Gender differences in fat metabolism. Curr Opin Clin Nutr Metab Care 4, 499-502.

32. Polotsky HN \& Polotsky AJ (2010) Metabolic implications of menopause. Semin Reprod Med 28, 426-434.

33. Romaguera D, Norat T, Vergnaud AC et al. (2010) Mediterranean dietary patterns and prospective weight change in participants of the EPIC-PANACEA project. Am J Clin Nutr 92, 912-921.

34. Mendez MA, Popkin BM, Jakszyn P et al. (2006) Adherence to a Mediterranean diet is associated with reduced 3-year incidence of obesity. J Nutr 136, 2934-2938.

35. Beunza JJ, Toledo E, Hu FB et al. (2010) Adherence to the Mediterranean diet, long-term weight change, and incident overweight or obesity: the Seguimiento Universidad de Navarra (SUN) cohort. Am J Clin Nutr $\mathbf{9 2}$, $1484-1493$.

36. Wareham NJ, van Sluijs EM \& Ekelund U (2005) Physical activity and obesity prevention: a review of the current evidence. Proc Nutr Soc 64, 229-247.

37. Slimani N, Fahey M, Welch AA et al. (2002) Diversity of dietary patterns observed in the European Prospective Investigation into Cancer and Nutrition (EPIC) project. Public Health Nutr 5, 1311-1328.

38. Agudo A, Slimani N, Ocké MC et al. (2002) Consumption of vegetables, fruit and other plant foods in the European Prospective Investigation into Cancer and Nutrition (EPIC) cohorts from 10 European countries. Public Health Nutr 5, 1179-1196. 